\title{
TES X-ray Maicrocalorimeters for X-ray Astronomy and Material Analysis
}

\author{
Kazuhisa Mitsuda \\ Institute of Space and Astronautical Science, Japan Aerospace Exploration Agency, 3-1-1 \\ Yoshinodai, Chuo-ku, Sagamihara, 252-5210, Japan
}

\begin{abstract}
TES X-ray microcalrimeter arrays provide not only high-energy resolution (FWHM

$<10 \mathrm{eV}$ ) in X-ray spectroscopy but also imaging and high-counting-rate capabilities. They are very promising spectrometer for X-ray astronomy and material analysis. In this paper, we report our recent progress. For material analysis, we have fabricated $8 \times 8$ format array with a fast signal response $(40 \mu \mathrm{s})$ and proved the energy resolution of $5.8 \mathrm{eV}$ FWHM at $5.9 \mathrm{keV}$. We developed common biasing scheme to reduce number of wirings from room temperature to the cryogenic stage. From measurements using the newly-designed common-bias SQUID array amplifier chips, and from numerical simulations, we demonstrated that signal cross talks due to the common bias is enough small. For space applications, we are developing frequency-division signal multiplexing system. We have fabricated a baseband feedback system and demonstrated that the noise added by the feedback system is about $4 \mathrm{eV}$ FWHM equivalent for $16 \mathrm{ch}$ multiplexing system. The digital to analog converter (DAC) dominates the noise, and needs be reduced by a factor of four for future astronomy missions.
\end{abstract}

Keywords: Microcalorimeters, Superconducting Transition Edge Sensors

(TES), X-rays astronomy, X-ray micro analysis

\section{Introduction}

An X-ray microcalorimeter detects individual X-ray photons as a heat input and determines the $\mathrm{X}$-ray energy from the increase of temperature [1]. The ideal

Preprint submitted to Physica C: Superconductivity and its Applications

March 28, 2016 
energy resolution of this detector is limited by the thermal fluctuation. When

5 it is operated at a cryogenic temperature of $100 \mathrm{mK}$, an FWHM (full width half maximum) resolution of a few $\mathrm{eV}$ can be obtained for soft X-rays of several $\mathrm{keV}$ with a realistic size of detector. This is by a factor of 50 better than the energy resolution of semiconductor detectors. The first generation X-ray microcalorimter uses a thermistor to sense the temperature increase, while the second generation, i.e. TES (transition edge sensor) microcalroimeter utilizes superconducting-transition-edge [2]. Advantages of TES microcalorimeters over thermistor-type calorimeters are two folds [3]. Firstly, a large format array of a few kilo pixels becomes possible even for space missions, where the cooling power is very limited (typically a few $\mu \mathrm{W}$ at $100 \mathrm{mK}$ ). Utilizing the low impedance of 15 TES device (a few $\mathrm{m} \Omega$ while it is $\sim 1 \mathrm{M} \Omega$ for thermistors), different methods for signal multiplexing at cryogenic-temperature stage are extensively studied in order to reduce number of wire harnesses between room temperature electronics and the detector. A large format array is essential for the focal plane detectors of future X-ray astronomy missions. Secondly, the time constant of thermistor calorimeters due to the strong electro-thermal feedback (ETF). After absorbing an X-ray photon, the device returns its equilibrium temperature with a certain time constant. For thermistor-type microcalorimeters, where the ETF is weak, the time constant is determined by the thermal time scale that is the

25 thermal capacity divided by the thermal conductance of the thermal link to the thermal bath. On the other hand, for TES microcalorimeters the heat from the $\mathrm{X}$-ray photon is compensated by the decrease of heat dissipation from the thermometer (TES). As a result the time constant becomes much shorter than the thermal time scale. This makes the maximum available counting rate higher by an order of magnitude. High counting rate is essential for ground applications, such as material analysis. We can also increase the maximum counting rate by using multiple pixels simultaneously.

Our group at ISAS JAXA is developing TES microcalorimeters for two purposes in collaboration with Kyushu University, NIMS, Hitachi HiTech Science, 
and Tokyo Metropolitan University. For material analysis, we are developing a sensor system for an energy dispersive X-ray spectrometer (EDX) for a scanning transmission electron microscope (STEM) $[4,5]$. The requirements for the system is energy range of 0.5 to $15 \mathrm{keV}$, an energy resolution of $<10 \mathrm{eV}$ at $6 \mathrm{keV}$, and a maximum counting rate of $20 \mathrm{kcps}$. In order to satisfy the high

40 counting rate requirement, we adopted an $8 \times 8$ format TES microcalorimeter array. In this system we do not need to multiplex the signal because the extremely-low-microvibration, cryogen-free dilution refrigerator [6] has enough cooling power to mount the low-power 64 SQUID (superconducting quantum interferometer device) array amplifies (SAA's) [7] on the cold stage. We developed common-biasing schemes both for the TES and SAA biases in order to reduce the number of wires from cold stage to the room temperature electronics by a factor of about two. The present status of the calorimeter array and the common-biasing schemes are reported in section 2 .

For X-ray astronomy space missions, our goal is $32 \times 32$ format $1 \mathrm{k}$-pixel array covering energy range of 0.1 to $2 \mathrm{keV}$ with a FWHM energy resolution of $\sim 2 \mathrm{eV}$. Combined with a short-focal length $(<2 \mathrm{~m})$ X-ray focusing optics, this device will detect and survey so-called missing Baryons, which believed to exit in a form of very low-density hot gas ( 10 times the critical density of the Universe and a $1 \mathrm{MK}$ temperature) in intergalactic space [8]. A key technology 55 to realize this system is signal multiplexing at a cryogenic temperature. We will describe the present status of the frequency division multiplexing (FDM) system in section 3 .

\section{Ground applications}

\subsection{Microcalorimeter array}

60 We designed and fabrication the calorimeter arrays in house [9]. The facilities in the RF nano-technology clean room of JAXA in Sagamihara Campus was mainly used for the fabrication. The sizes of the TES, the X-ray absorber, and the membrane for thermal-link are, respectively, $180 \times 180 \mu \mathrm{m}^{2}, 120 \times 120 \mu \mathrm{m}^{2}$, 
and $300 \times 300 \mu \mathrm{m}^{2}$. The pixels are placed in an $8 \times 8$ format with a $390 \mu \mathrm{m}$ pitch.

We use aluminum superconducting electrodes whose wiring spacing and line width are both $10 \mu \mathrm{m}$. The thickness of the absorber $(\mathrm{Au})$, the TES (Ti/Au), and the thermal-link membrane $\left(\mathrm{SiN}_{x} / \mathrm{SiO}_{2}\right)$ are, respectively, $2.5 \mu \mathrm{m}, 40 / 90$ $\mathrm{nm}$, and 1.0/0.5 $\mu \mathrm{m}$. The transition temperature of the TES was designed to be $200 \mathrm{mK}$, which is the major difference of the design from that for space applications.

The saturation energy, with which the TES goes beyond the transition edge, is one of the major design parameters. It is roughly estimate with $E_{\mathrm{sat}}=C T / \alpha$, where $C$ and $T$ are respectively the heat capacity and the operation temperature of the device, and $\alpha=d \ln R / d \ln T$ is the sensitivity of the TES. Then the ideal energy resolution and the decay time constant of X-ray pulses are respectively given by,

$$
\begin{gathered}
\Delta E=\xi \sqrt{E_{\mathrm{sat}} T}, \\
\tau=\frac{E_{\mathrm{sat}}}{G T},
\end{gathered}
$$

where $\xi$ is a constant and $G$ is the thermal conductance of the thermal link to the heat bath. In ground applications, acceptance of a high counting rate is one of the important requirement. For this purpose, high $T$ value is preferred (Eq. (2). However, this will degrades the energy resolution (Eq. (1)). Thus compromise between the maximum counting rate and the energy resolution must be made. For the present ground applications, where the energy resolution requirement is not very stringent, we selected $200 \mathrm{mK}$ and obtained a short decay time constant of $40 \mu \mathrm{s}$. In Fig. 1 we show the photographs of the device and the pulse height spectrum obtained for $\mathrm{Mn} \mathrm{K}$ lines from ${ }^{55} \mathrm{Fe}$ isotope illuminated on one of the pixels. A FWHM energy resolution of $5.8 \mathrm{eV}$ was obtained.

\subsection{Readout method: Common biasing scheme}

If all the 64 TES's and 64 SAA's are separately connected with the room temperature electronics, we need 512 pairs. This is a very large number even for the ground cooling system. We, therefore, developed common bias schemes 
for the TES and SAA, respectively. We group 64 pixels into 8 subgroups. Then 8 TES 's and 8 SAA' s from a subgroup are, respectively, biased with a single bias line from room-temperature electronics. This reduces number of wires from

95512 to 288. Two different configurations of common bias, series and parallel, may be considered. From a simple analytic calculations, we find that the cross talk between different pixels is much smaller for series configuration both for TES and SAA [10]. The amplitude of the cross talk depends on the ratio of the TES resistance to the bias shunt resistance $\left(R_{\mathrm{S}}\right)$ for TES (see Fig. $\left.2(\mathrm{a})\right)$ and ratio of dynamic resistance of SAA $\left(R_{\mathrm{dyn}}\right)$ to the SAA bias resistance $\left(R_{\mathrm{B}, \mathrm{SAA}}\right)$ divided by the loop gain of the flux-locked loop (FLL). Assuming our typical parameters, the amplitude of cross talk is estimated to be $\sim 10^{-7}$ for TES bias and $10^{-3}$ for SAA bias, respectively. We then estimated the effect on the energy resolution by numerical simulations in which the random occurrence of X-ray events is taken into account. The results show that the degradation of energy resolution is at most $10^{-4}$ of the pulse height. Thus it is small enough $(<2 \mathrm{eV})$ even with a cross talk by $15 \mathrm{keV}$ photons.

We designed a common-bias SAA chip on which eight SAA's are connected in the series common-bias configuration. Then chips were fabricated by the CRAVITY facility of AIST in Tsukuba (Fig. 2 (b)). We measured the crosstalk by feeding a dummy X-ray pulse signal to one of the SAA and measuring the outputs of other SAA' s. We found that the amplitude is about $10^{-3}$, which is very consistent with the above estimation (Fig. 2 (c)). We then connected the common-bias SAA chip to the $8 \times 8$ format TES microcalorimeter and confirmed that we can read the microcalorimeter signals simultaneously with this method.

\section{Space applications}

We have fabricated up to a $16 \times 16$ format array [11]. Our present goal is $32 \times 32$. With the smaller cooling power of the space cooling system, the common bias scheme will not work. Thus we need signal multiplexing at a cryogenic temperature stage. 
Frequency division multiplex utilizes the difference in frequency band widths of SAA's (<5 MHz when operated with high-frequency FLL electronics) and TES signals $(<10 \mathrm{kHz})[12]$. TES' $\mathrm{s}$ are biased with AC current of different frequencies and summed together at the input coil of SAA (Fig. 3 (a)). Because of long $(>1 \mathrm{~m})$ wire harness between the cold stage and the room temperature electronics, a significant phase shift occurs between the SAA and the low noise amplifier, which makes the FLL feedback unstable when AC bias of $\sim 1 \mathrm{MHz}$ or higher frequencies are used. Baseband feedback [13] is a scheme to avoid this instability by correcting for the phase shift at the carrier frequency. We developed a SAA for 4-channel FDM (Fig. 3 (b)), and a digital baseband feedback system using fast ADC (analog to digital converter), FPGA (field programmable gate array), and DAC (digital analog convert) [14]. In Fig. 4, we show some of the performances of the digital baseband feedback system. Panel (c) shows the noise expected when the system is expanded up to 16 channels. If the number of channel is larger than or equal to $\sim 8$, the noise level of the read-out system will be dominated by that of DAC. The noise equivalent to the LNA input is estimated to be $22 \mathrm{nV} / \sqrt{ } \mathrm{Hz}$ when 16 signals are multiplexed. Contribution to the energy resolution is estimated to be $\sim 4 \mathrm{eV}$ for the $\mathrm{X}$-ray microcalorimeters described in section 2.1. Thus, although the noise level is not very high, it needs be reduced by a factor of about 4 for future space missions.

This system was connected to four pixels of the TES microcalorimeters described in section 2.1. Fig. 4 (a) show the frequency response of the LC filters, and (b) shows the time history of X-ray pulse records. Although we successfully multiplexed signals from 4 TESs, we failed to obtain an energy resolution expected from the DC bias performance of the TES microcarolimeter and the noise level estimation of the readout system. We consider this was due to experimental setup, where cross talk between the TES bias lines and SAA output signal lines were significantly high. 


\section{Acknowledgements}

150 gayoshi, Mr. R. Yamamoto, Mr. A. Chiba, Mr. T. Hayashi, Ms. H. Muramatsu, Mr. K. Maehisa, and Dr. Y. Takei (ISAS, JAXA), Dr. K. Maehata, Dr. N. Iyomoto, and Mr. A. Takano (Kyushu Univ.), Dr. T. Hara (NIMS), Dr. M. Hidaka, Dr. S. Nagasawa, Dr. S. Kojiro (AIST), Dr. K. Tanaka (Hitach HS), and Dr. Y. Yamanaka (TNS). The results presented in this paper are based

on the results obtained with collaboration with those people. The TES microcalorimeters were fabricated using the Ti/Au sputter machine at TMU and the micro/nano fabrication facility at ISAS, JAXA. The author is grateful to the Prof, T. Ohashi (Tokyo Metro. U.), and Prof. K. Kawasaki and Mr. A. Miyachi (ISAS, JAXA) for the maintenance of the facilities. This work was partly supported by SENTAN, Japan Science and Technology Agency (JST) and JSPS KAKENHI Grant Number 80183961.

\section{References}

[1] S. H. Moseley, J. C. Mather, D. McCammon, , J. Appl. Phys 56 (1984) 1257.

[2] K. D. Irwin, J. C. Mather, D. McCammon:, , Appl. Phys. Lett 66 (1995) 1998.

[3] K. D. Irwin, G. C. Hilton, Transition-Edge Sensors, in Cryogenic Particle Detection, ed. by C. Enss, Topics in Applied Physics Volume 99, Springer, 2005.

[4] T. Hara, Tanaka, K. K, Maehata, K. Mitsuda, N. Y. Yamasaki, M. Ohsaki, K. Watanabe, X. Yu, T. Ito, Y. Yamanaka, , J. of Electron Microscopy 59 (2010) 17, 10.1093/jmicro/dfp043.

[5] K. Maehata, T. Hara, K. Mitsuda, M. Hidaka, K. Tanaka, Y. Yamanaka, , J. of Low Temp. Physics submitted. 
[6] K. Maehata, N. Iyomoto, Y. Yamanaka, T. Ito, T. Hara, K. Mitsuda, N. Y. Yamasaki, K. Tanaka, , J. Supercond Nov. Magn 28 (2015) 1161.

[7] K. Sakai, Y. Takei, R. Yamamoto, N. Y. Yamasaki, K. Mitsuda, M. Hidaka, S. Nagasawa, S. Kohjiro, T. Miyazaki, , J. of Low Temp. Physics 176 (2014) 400, 10.1007/s10909-013-1040-1.

[8] Y. Suto, K. Yoshikawa, N. Yamasaki, K. Mitsuda, R. Fujimoto, T. Furusho, T. Ohashi, M. Ishida, S. Sasaki, Y. Ishisaki, Y. Tawara, A. Furuzawa, , J. of Korean Physical Society 45 (2004) S110.

[9] H. Muramatsu, K. Nagayoshi, T. Hayashi, K. Sakai, R. Yamamoto, K. Mitsuda, N. Y. Yamasaki, K. Maehata, T. Hara, , J. of Low Temp. Physics submitted.

[10] R. Yamamoto, K. Sakai, K. Maehisa, K. Nagayoshi, T. Hayashi, H. Muramatsu, Y. Nakashima, K. Mitsuda, N. Y. Yamasaki, Y. Takei, M. Hidaka, S. Nagasawa, K. Maehata, T. Hara,, J. of Low Temp. Physics submitted.

[11] U. Morita, Y. Yamakawa, T. Fujimori, Y. Ishisaki, T. Ohashi, Y. Takei, K. Yoshida, T. Yoshino, K. Mitsuda, N. Y. Yamasaki, R. Fujimoto, H. Sato, Y. Minoura, N. Takahashi, T. Homma, S. Shoji, Y. Kuroda, M. Onishi, , NIM-A 559 (2006) 539, 10.1016/j.nima.2005.12.064.

[12] T. Miyazaki, M. Yamazaki, K. Futamoto, K. Mitsuda, R. Fujimoto, N. Iyomoto, T. Oshima, D. Audley, Y. Ishisaki, T. Kagei, T. Ohashi, N. Yamasaki, S. Shoji, H. Kudo, Y. Yokoyama, , in: Low Temperature Detectors, ed. by F.S. Porter, et al. American Institute of Physics, 2002.

[13] J. van der Kuur, J. Beyer, D. Boersma, M. Bruin, L. Gottardi, R. den Hartog, H. Hoevers, R. Hou, M. Kiviranta, P. J. de Korte, B. J. van Leeuwen, , in: LTD13. AIP Conference Proceedings, 2009, p. 245.

[14] K. Sakai, R. Yamamoto, Y. Takei, K. Mitsuda, N. Yamasaki, M. Hidaka, S. Nagasawa, S. Kohjiro, T. Miyazaki, , J. of Low Temp. Physics, submitted. 


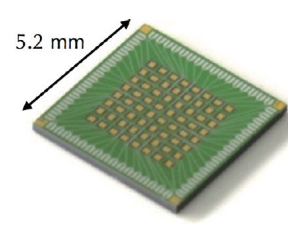

(a)

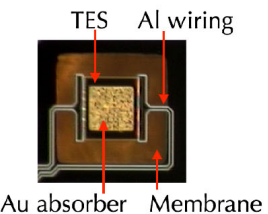

(b)

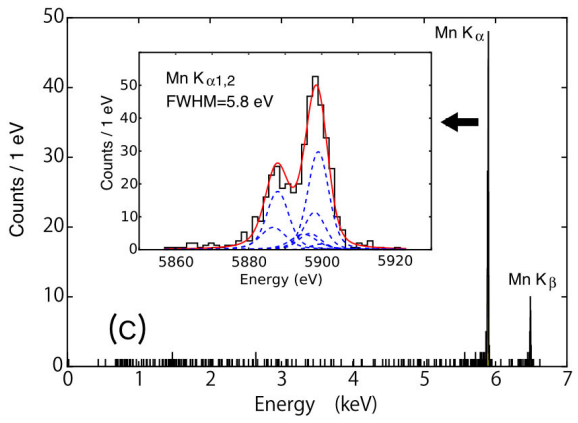

Figure 1: (a) $8 \times 8$-format TES-microcalorimeter-array chip; (b) close up photo of a pixel; (c) pulse height spectrum of ${ }^{55} \mathrm{Fe}$ radio isotope.

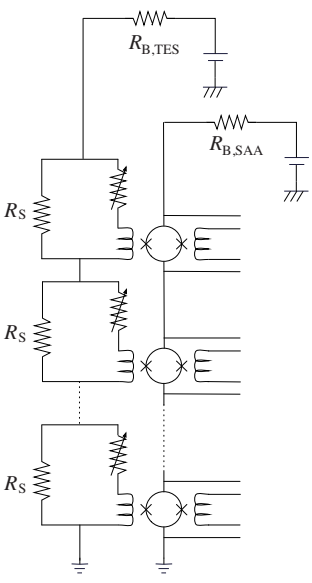

(a)

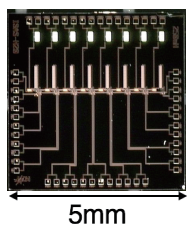

(b)

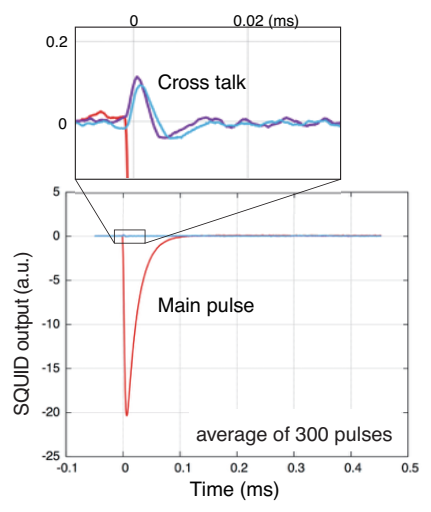

(c)

Figure 2: (a) Common biasing scheme for TES and SAA biases. SAA is described as a single SQUID for simplicity, although it is 16 series of SQUIDs; (b) Common-bias SAA chip for 8 channels; (c) SQUID outputs of the channel to which dummy pulse signals were fed, and of its adjacent and the next adjacent channels. The top panel shows the expansion around the rise of the dummy pulse. 


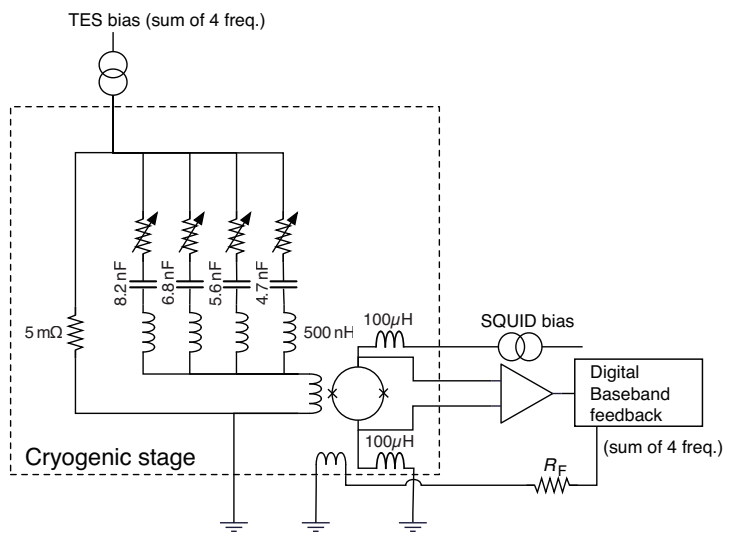

(a)

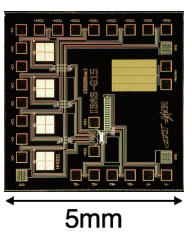

(b)

Figure 3: (a) Concept of frequency division multiplexing (FDM); (b) SAA chip for FDM. It contains four inductors for four TES inputs. 


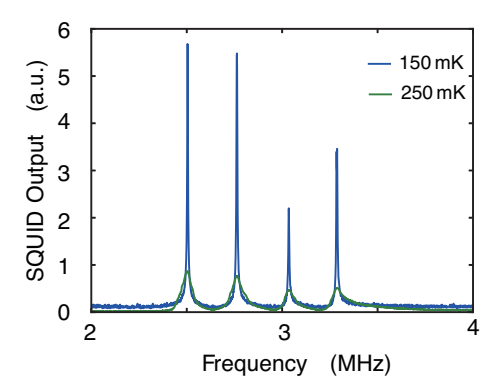

(a)

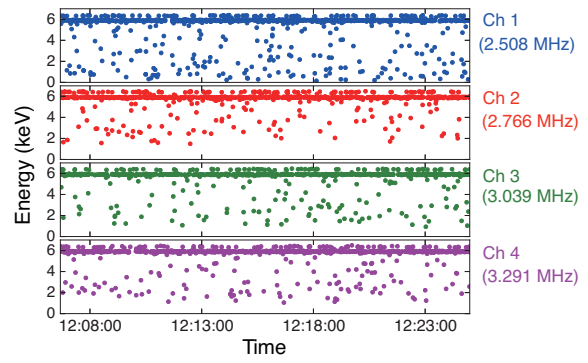

(b)
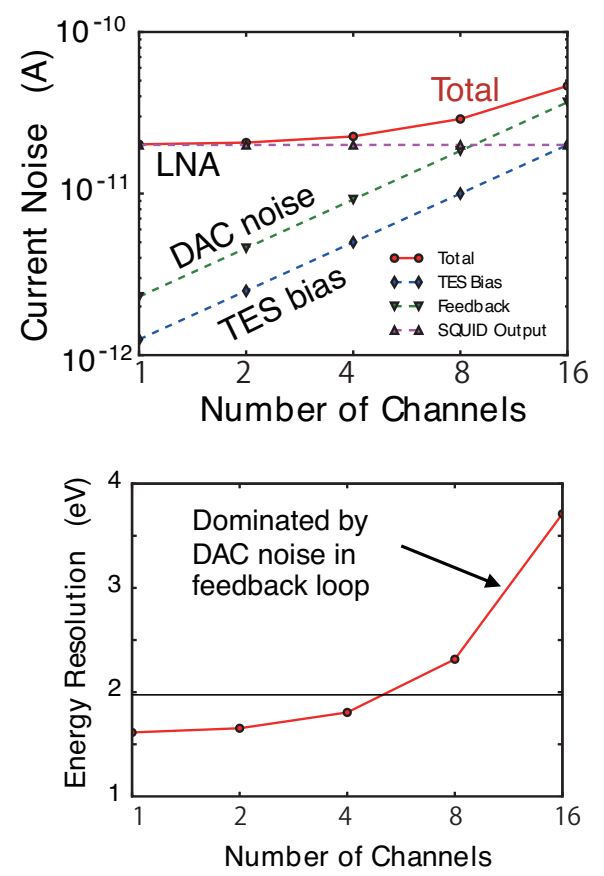

(c)

Figure 4: (a) Measured frequency response of the LC filters for 4 ch FDM; (b) X-ray event records of 4-channel simultaneous readout using FDM; (c) noise estimation of the FDM signal processing system. 